\title{
Éditorial
}

\section{La publicité des fumées d'usine ou le temps des pollutions bienfaisantes}

\author{
Serge Lambert* \\ Expert environnement/écologue
}

\footnotetext{
$\checkmark$
}

En ce temps-là, et il n'y a pas si longtemps, noires fumées d'usine n'étaient pas vice. Bien au contraire, patrons d'usines, ouvriers, cadres et populations qui en vivaient, étaient fiers de ces hautes et nombreuses cheminées laissant échapper, chaque heure, chaque jour, de lourds panaches blancs, gris ou noirs. Et tous, bénéficiaient de leurs odeurs acres, piquantes, toxiques parfois, mais qui le savait?

Certes la ménagère, ma tante, femme de " mineur 》 à Moyeuvre-Grande près de Metz, n'appréciait pas toujours d'avoir à relaver linges et draps couverts de suie ou de moucherons... Mais la vie près d'une usine était ainsi. Quant à aller se plaindre à l'usine, aucune femme n'y songerait. Pensez donc, son homme, ses fils, oncles et neveux bien sûr y travaillaient; elle-même n'y faisait-elle pas quelques «ménages » de bureau!

Tout au contraire, ces hautes cheminées et leurs lourds panaches noirs étaient, pour ceux qui y travaillaient ou en vivaient, symbole d'emploi, de salaire, de mieux être ; pour l'industriel, le patron celui de la réussite sociale, du pouvoir, de la puissance.

L'absence de fumée, le silence qui l'accompagnait souvent devenaient soucis, anxiété, drame à venir. Et finalement les destructions de cheminées (comme celles des usines) ont été souvent vécues comme des morts, des assassinats, la fin ou l'interruption d'une vie de travail... la fin d'un monde.

Qui, aujourd'hui, oserait afficher, sur en-tête de lettre ou publicité qu'une fumée d'usine est non seulement innocente mais aussi symbole affiché et bien visible d'emploi, de prospérité ? Et pourtant au début de ce siècle, en pleine expansion industrielle et commerciale l'iconographie industrielle fait un usage généralisé, comme publicité ou en-tête de lettres, de photos d'usines, de bâtiments industriels et commerçants, toujours accompagnées de hautes cheminées fumantes.

A titre de premier exemple : l'industriel Gillet, bien connu des chimistes Lyonnais, créateur de la société Progil, ellemême, plus tard, composante de Rhône-Poulenc. En 190I, il engage $M$. Berthet. Plus précisément, écrit-il, « nous acceptons votre entrée dans notre maison à titre de voyageur pour l'exportation ". II devra, pour $250 \mathrm{~F} / \mathrm{mois}$ "visiter et inspecter 》 les postes commerciaux de l'Indochine, du Tonkin et Madagascar. II est intéressé au " chiffre net payé " mais sera lourdement sanctionné en cas de mauvais paiement ; « nous retrancherons de la somme totale des affaires du rayon, non pas seulement le montant net de cette mauvaise affaire mais le triple de ce montant ». De plus "nous n'acceptons aucune responsabilité à votre sujet et il est entendu qu'il n'y a aucun engagement réciproque entre nous, au sujet de la durée de votre emploi dans notre maison »! Moins qu'un CDD finalement. On notera un en-tête de lettre qu'on ne peut plus simple «Gillet et Fils Villeurbanne (Rhône) Teinture Impression Apprêt »

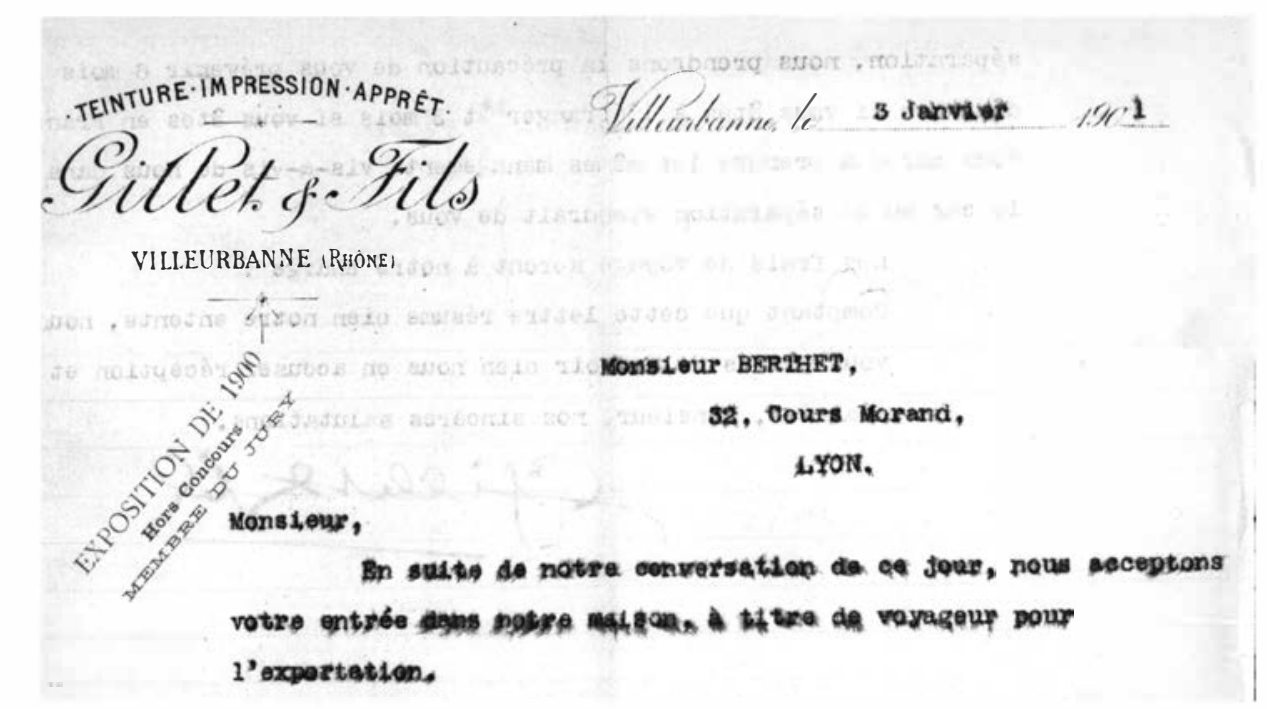


Deux années plus tard en 1903, M. Gillet écrit à nouveau à M. Berthet'. Entre temps, M. Gillet industriel avisé, com-

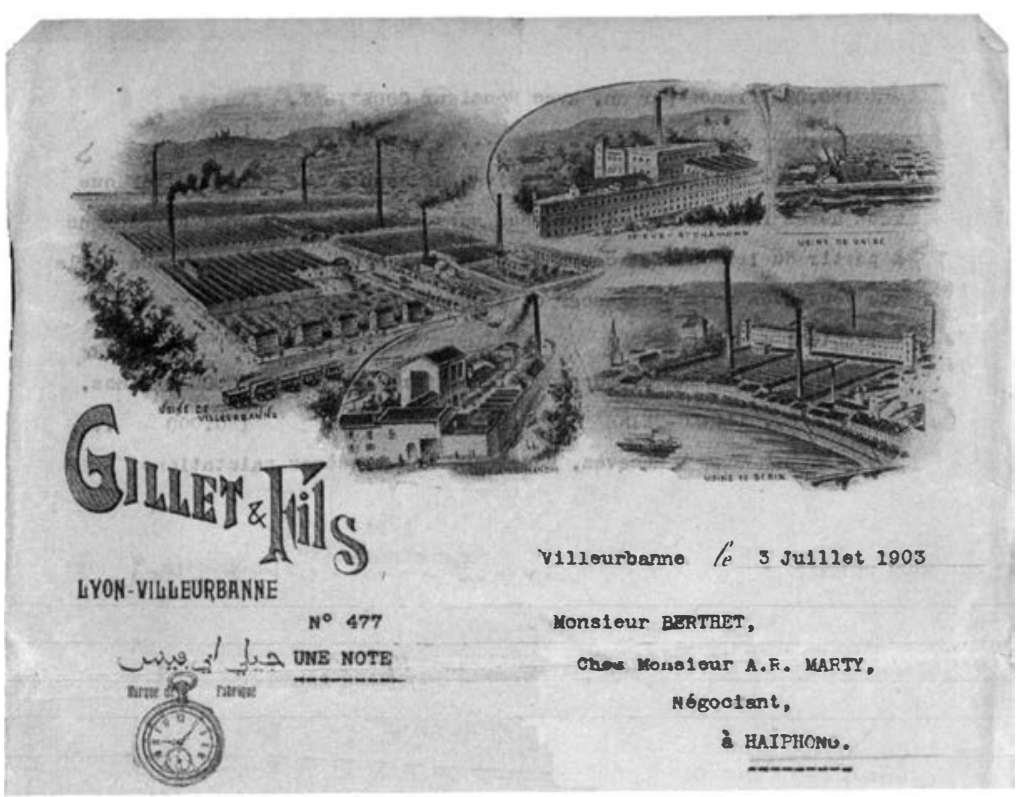

pétent, exigeant de lui-même et de son personnel, a fait de bonnes affaires et a étendu son empire. II se trouve, à Lyon, rapidement à la tête de plusieurs usines. On le fait savoir et l'en-tête de ses lettres est totalement transformé, M. Gillet et ses Fils ont représenté les cinq usines qu'ils possèdent à Lyon et dans ses environs. La puissance se décline par la surface des bâtiments mais aussi par le nombre de cheminées qui toutes, fument, fument en de longs panaches noirs parfaitement authentiques, (leur forme est celle qu'on observe toujours en pareil cas). Mes usines sont bien là et elles travaillent, semble dire M. Gillet, pour preuve les cheminées fument... Finalement publicité efficace, sans en faire, pas de logos recherchés ou artificiels, le concret du travail qui se voit, et souvent de loin. Des cheminées et des fumées à ne plus savoir qu'en faire...
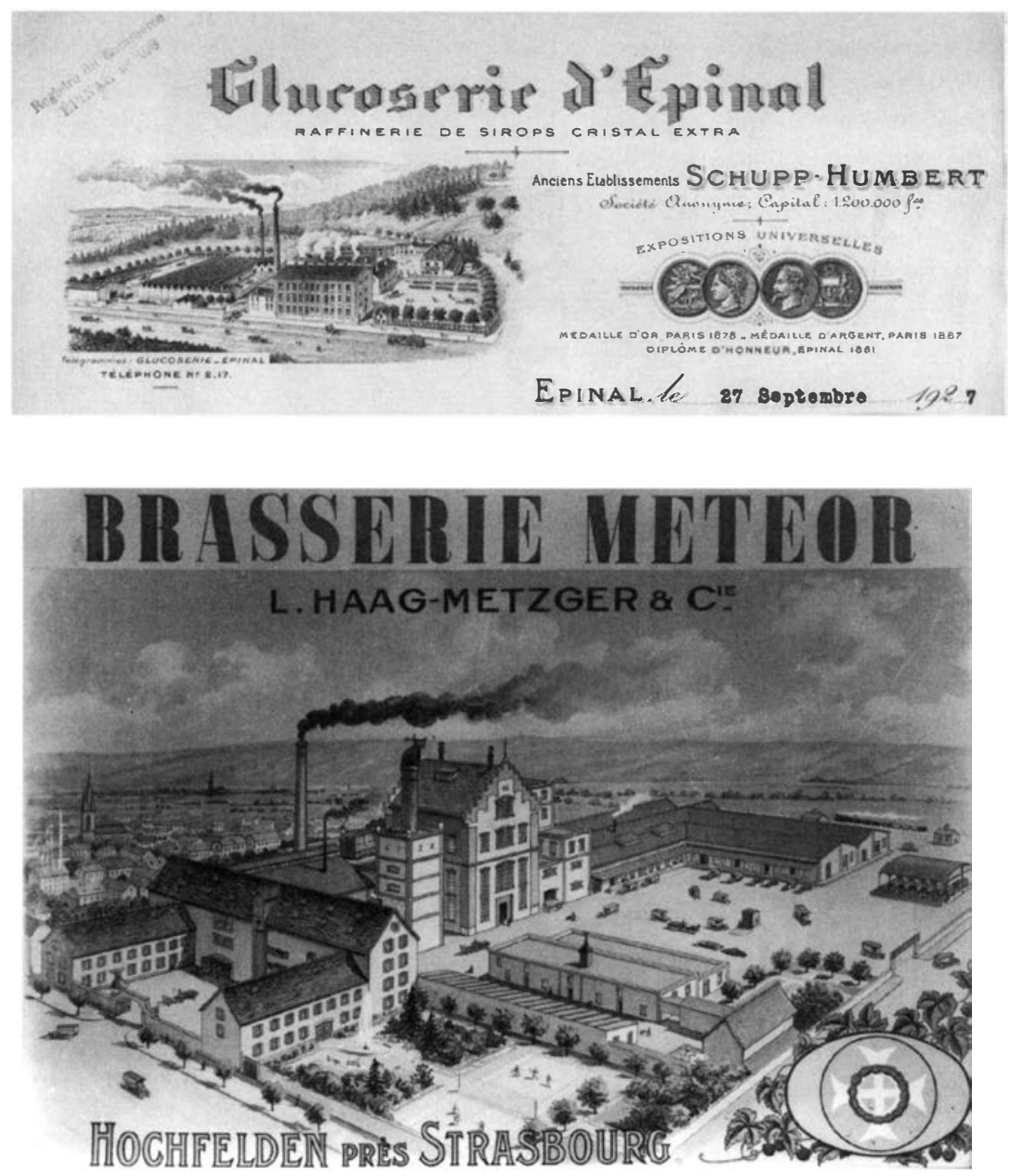
Et ce mode d'iconographie industrielle est commun à tous, industriels ou commerçants, négociants : en-tête de lettres et publicités avec reproduction de la trilogie usines-cheminées-fumées. Mieux encore, les bâtiments à simple vocation commerciale n'échappent pas à la règle. Qu'on en juge par ce négociant en vin de Bordeaux qui choisit très certainement une photo prise en hiver (ou retouchée) pour que sa cheminée, bien modeste certes, affiche toutefois, une fumée...active! sifflements, des plaintes haletantes, des grondements formidables !

Ces hautes cheminées fumantes, clochers silencieux mais bien visibles de l'industrie, avaient leur légitimité directe, éloigner, disperser la pollution, et indirecte, travail et salaire. Et c'était surtout cette vision dernière qu'on retenait. Qu'on en juge avec ce slogan souvent cité dans les cités ouvrières du Nord Est de la France et dont on fera notre conclusion: "Quand la cheminée fume à l'usine la soupe fume à la cuisine».

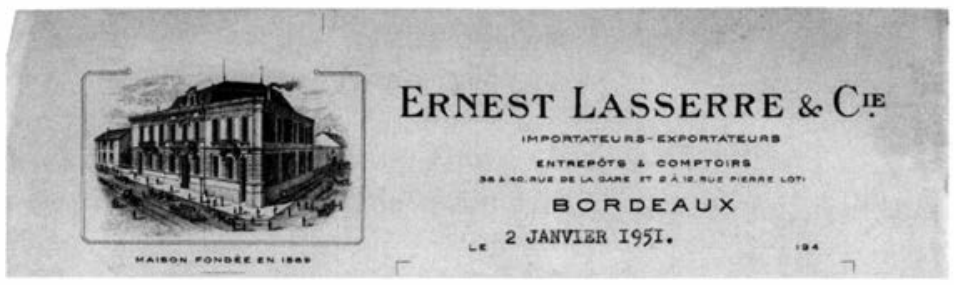

\section{Serge Lambert}

Expert environnement/écologue - 2, allée des Cèdres - 69370 Saint-Didier-au-Mont-d'Or

Les manuels scolaires n'hésitent pas à grossir le trait pour une démonstration efficace. Un dessin, censé représenter les usines du Creusot et extrait du « Tour de la France par deux enfants » de G. Bruno (I900, page III), nous montre des cheminées avec panaches monstrueux, manifestement exagérés. Le texte d'accompagnement est à leur mesure, Le Creusot y est décrit à la fois, comme puissance " qui effraie » et " bienfaisante qui travaille pour l'humanité » mais qui ressemble à un "grand incendie », une " immense fournaise » et où on entend des

Note:

J'ai très fortuitement avec une lettre semblable à celles évoquées ci dessus, retrouvé la «trace » de $M$. Berthet $M$. En 1912, dix années plus tard, il habite de nouveau à Lyon mais a quitté la « maison Gillet » pour alors, démarcher pour le compte de la «maison » Boissiere fils et Cie ; manufacture de blanchiment, teinture et impression. Bien entendu leur usine à Notre-Dame-de-Bondeville peut, elle aussi, s'enorgueillir de trois belles et hautes cheminées, " arborant " chacune un long panache de fumée!

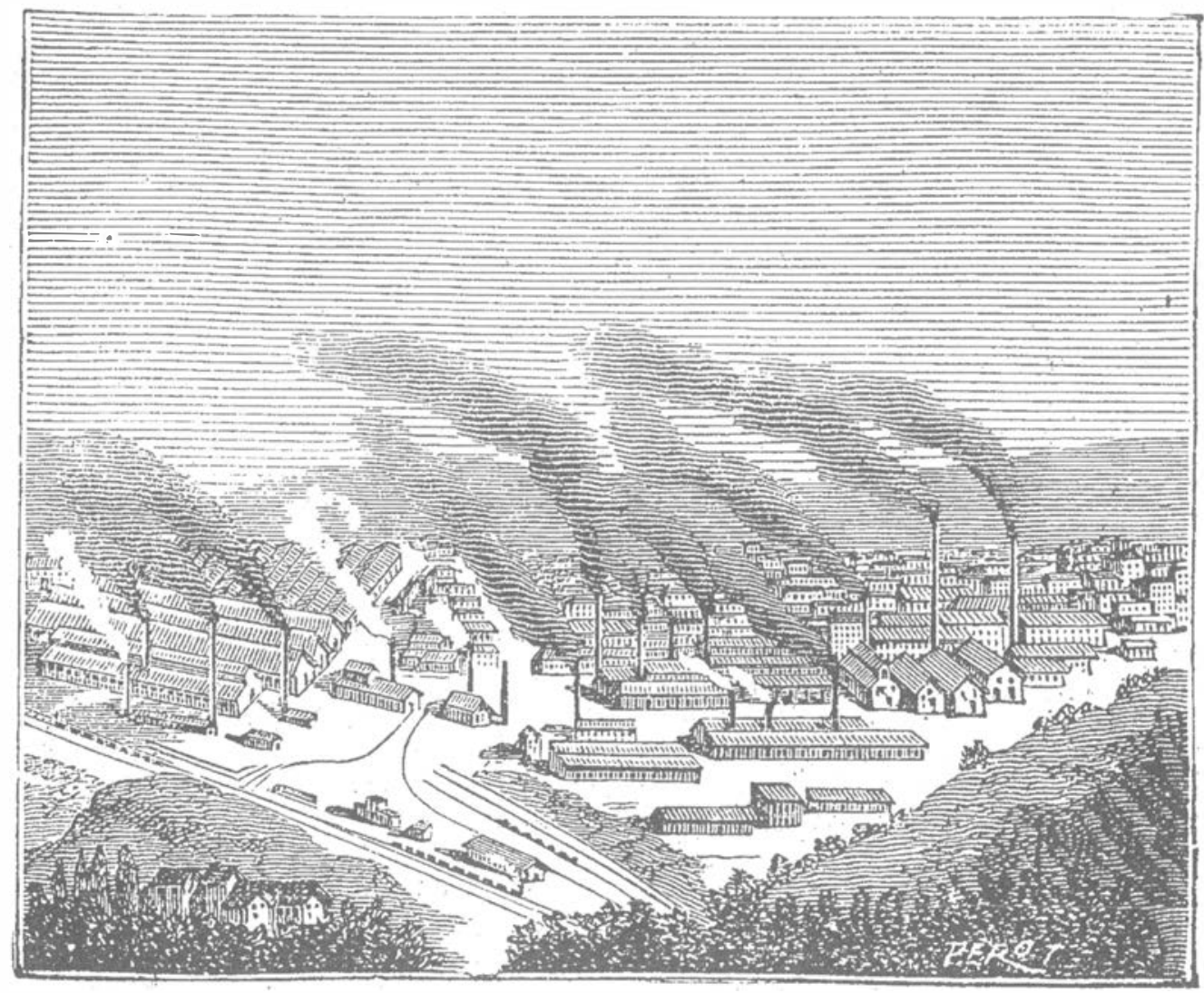

